\title{
ARTICULACIÓN DE LA EDUCACIÓN MEDIA CON EL MUNDO PRODUCTIVO
}

\author{
JULIA EDITH CERQUERA OLAYA
}

日 presente trabajo de investigación estuvo motivado por la necesidad de determinar el grado de cumplimiento de la política pública "ARTICULACIÓN DE LA EDUCACIONN CON E MUNDO PRODUCTIVO- LA FORMACIÓN DE COMPETENCIAS LABORALES" reflejada en el convenio interadministrativo 002 de 2005 suscrito entre la Secretaria de Educación de Neiva y el Servicio Nacional de Aprendizaje SENA, han cumplido con los propósitos señalados en cada uno de ellos, como estrategia de contextualización. Con el fin de ofrecer a los lectores un punto de partida para la lectura y comprensión del presente artículo, en el cuadro No 1 se presenta la relación que existe entre los objetivos de la política pública y el convenio interadministrativo 002 de 2005.

\section{CuadroNo.1}

OBJETIVOS DE LA POLITICA PUBLICA 52 YEL CONVENIO INTERADMINISTRATIVO 002 DE 2005 s.

\section{OBJETNOS DE LA POLITTICA PUBLICA}

a) Contribuir al desarrollo de competencias taborates generales en los estudiantes de la educación bấsica y media para facilitar su vinculación activa a la vida productiva.

b) Promover alternativas de formación de competencias laborales en los estudiantes de la educación media mediante ed acercamiento de las instituciones educativas al mundo productivo.

c) Me jorar la calidad de la oferta orientada a la formación laboral específica de los estudiantes de la educación media mediante procesos de acreditación de programas.

\section{OBJETTVOS DEL CONVENIO INTERADMINISTRATIVO 002 DE 2005}

\section{OBJETIVO GENERAL:}

Desarrollar el programa de Articulación con las instituciones de educación media técnica estatales, con el propósito de contribuir a la calidad de la formación técnica del país y facilitar el mejoramiento continuo de los estudiantes del grados 10 y 11.

\section{OBJETIVOS ESPECÍFICOS:}

1. Ofrecer formación técnica que proporcione posibilidades de movilidad hacia otras modalidades y niveles educativos (cadena de formación) o para vincularse laboralmente;

2. Ejecutar acciones de actualización pedagógica y técnica para el mejoramiento continuo de los docentes de las instituciones vinculadas al programa de articulación;

3. Asimilar por las instituciones vinculadas, los programas que ofrece el SENA;

4. Elevar el nivel educativo de los jóvenes y facilitarles el acceso a estudios superiores;

5. Fomentar en los jóvenes las aptitudes empresariales y propiciar sus prácticas laborales; ó Propiciar la inserción de los jóvenes al trabajo;

7. Mejorar la oferta de la educación técnica y hacerla pertinente y de calidad de acuerdo a las exigencias del sector productivo.

\footnotetext{
52 COLOMBAA MINSTERIO DE EDUCAOON NAGONAL POFltica PÜblica, articulaciôn de la educación con el Mundo produrcllvo-La formadión décompetencias laborales, 2003, Disporible en Internet: http:/wwwmineducacion,gov co/1621/articks $85 / 77$ _ardhivo_pd pdr

53 SENA, Secretaria de Educaclón de Neiva, convenlo Enteradministrativo 0022005.
} 
En la coordinación del Programa de articulación de la educación media en el Centro de la Industria, la Empresa y los Servicios del SENA Neiva, se tienen la duda sobre el impacto que se estaría generando en la vida de los egresados que hicieron parte del proceso, puesto que los datos de cobertura eran fáciles de identificar a travếs de la información que se maneja en los aplicativos del SENA, pero no así para variables de impacto y la caracterización de los egresados; surge entonces los interrogantes:

¿Cuál ha sido el impacto del Convenio interadministrativo 002 de 2005 suscrito entre la Secretaría de Educación del municipio de Neiva y el SENA, en los jóvenes egresados de los programas Técnicos Laborales impartidos en las instituciones educativas Oliverio Lara, E Limonar y Humberto Tafur Charry de Neiva, durante el periodo 2008-2011?.

¿En qué tipo de actividades participan los egresados de los programas técnicos impartidos en las Instituciones Educativas ob jeto del proyecto?.

¿Cuál ha sido la contribución de la formación laboral ofrecida por las Instituciones Educativas, respecto a la actividad queen la actualidad desarrollan los egresados?

¿Continuaron los egresados su proceso de formación en los niveles de educación superiory cuántos relacionados con la formación técnica que recibieron en la ar ticulación?

¿Cuál es la percepción de los empleadores sobre las competencias de los egresados en el desarro llo de los trabajos asignados, en el caso de aquellos que se han vinculado al sector productivo?

¿Cuáles son los criterios que se deben tener en cuenta al momento de decidir la articulación y la elección de un programa Técnico Laboral por parte de las Instituciones Educativas? Los anteriores interrogantes fueron desarrollados en el trabajo de investigación denominado: "IMPACTO DEL PROCESO DE ARTICULACIÓN DE LA EDUCACIÓN MEDIA, SENA SECRETARIA DE EDUCACIÓN DE NENA, EN LOS EGRESADOS DE LAS INSTITUCIONES EDUCATNAS OUVERIO LARA, B LIMONAR Y HUMBERTO TAFUR CHARRY 2008-2017'

\section{Cuyos objetivo General fué:}

Establecer el impacto del proceso de articulación de la Educación Media, en los egresados de los programas articulados en las Instituciones Educativas Oliverio Lara Borrero, El Limonar y Humber to Tafur Charry, en el marco del convenio Interadministrativo 002 de 2005 suscrito entre la Secretaría de Educación de Neiva y el SENA. Periodo comprendido entre los años 2008-2011

Para el logro de este objetivo, se platearon los siguientes objetivos especificos.

- Caracterizar los aspectos socioeconómicos de los jóvenes egresados de las Instituciones Educativas Oliverio Lara Borrero, El Limonar y Humberto Tafur Charry de Neiva.

- Determinar el tipo de actividad que realizan los egresados de los programas técnicos impartidos.

- Identificar los egresados que continuarón la cadena de formación o educación superior relacionada con el oficio que aprendió. 
- Establecer k contribución de la formación técnica, en el desarrollo de las actividades que en la actualidad realizan los egresados.

- Determinar el nivel de satisfacción de los egresados con relación a la formación recibida.

- Determinar el concepto de los empleadores sobre las competencias de los egresados de los programas técnicos en articulación.

- Proponer ajustes al proceso para mejorar la puesta en marcha de la política.

Para cumplir con los propósitos del trabajo de investigación se tomó una muestra de 69 Jóvenes egresados de los programas de Cocina, Registro de Información contable y Financiera, Programación de Software y Sistemas, de las I.E. Oliverio Lara, El Limonar y Humberto Tafur Charry; a quienes se les aplicó un cuestionario para determinar su caracterización socioeconómica y el tipo de actividad que realizaron una vez terminaron su formación técnica en articulación con la educación media. Se realizó la revisión de documentos como los PEI y los programas de formación que el SENA ha articulado con la educación media en las 3 instituciones educativas señaladas, de igual manera se realizaron entrevistas a funcionarios de la Secretaría de Educación de Neiva, rectores, empresarios que ha contratado a egresados, con el fin de establecer la percepción frente al desarrolio del Programa.

La información recolectada refleja los siguientes resultados

\section{EDAD Y EMPLEO}

La edad promedio en que terminan el bachilleratos los jóvenes es de 16 a 17 años, pues tan solo el $9 \%$ culmina sus estudios de la media a los 18 anôos, tal como se observa en la gráfica No.1

Grafica No. 1. Edad de los egresados de las Instituciones Educativas de las Instituciones Educativas Objeto de estudio

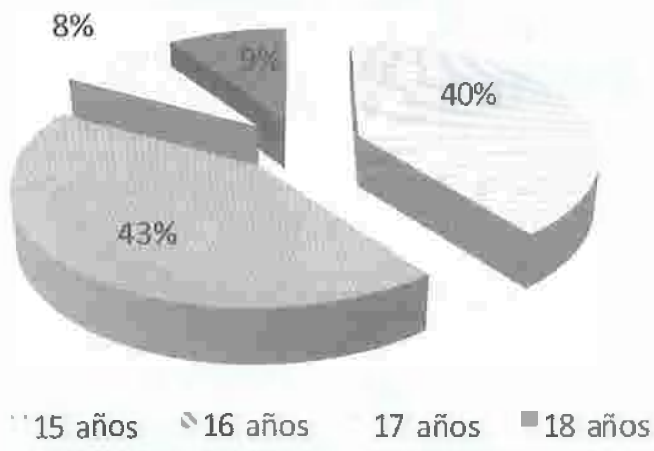

Fuente: Elaboración propia con base en ias fesult ados dei cuestionario aplicadoa egoresados de bs LE. objeto de estudio

La corta edad en que terminan los jóvenes no permite que estos egresados puedan desarrollar un trabajo en las condiciones que la Ley lo exige; deben presentar un permiso de trabajo tal como se expresa en la Sentencia C-215 de Marzo del 200754. Esta premisa debe tenerse en cuenta frente a la pretensión del objetivo específico del acuerdo 002 suscrito entre la Secretaría de Educación de Neiva y el SENA: "Ofrecer formación técnica que proporcione

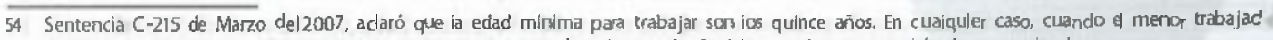
tenga entre 15 y 17 años, debę soilcitar permiso a Ministerio de i Protección Social para iaborar ai servicio de un empleador. 
posibilidades de movilidad hacia otras modalidades y niveles educativos (cadena de formación) o para vincularse laboralmente".

Por otra parte es importante tener en cuenta que la política pública "ARTICULACIÓN DE LA EDUCACIÓN CON EI MUNDO PRODUCTIVO LA FORMACIÓN DE COMPETENCIAS LABORALES" considera que la formación laboral, aumenta en los estudiantes la posibilidad de conseguir un empleo, lo que sería posible, si el aparato productivo tuviera las condiciones para absorber la mano de obra calificada (en edad laboral) de los jóvenes egresados del bachillerato ode las universidades, pues lo que señalan las estadisticas es queen Colombia el $47 \%$ de los desempleados son jóvenes, es decir, hay 1.260 .000 jóvenes sin ocupación remunerada. Incluso durante la mejor época de la economía del pás, el año 2007, cuando la tasa de desempleo general se ubicó en un $10.3 \%$, para los jóvenes fue de $20.30 \%$. Esta situación ejerce presión aún más fuerte sobre las mujeres jóvenes. El 26.3\% de ellas no devengan un salario 55.

\section{INGRESOS FAMILIARES}

De acuerdo con la información de la ficha de matrícula de los aprendices se establece que el $97 \%$ de los egresados estấn en los estratos 1 y 2 y que sólo el $3 \%$ pertenece al estrato tres. Esto está en concordancia con lo que se puede observar en la Gráfica 2, respecto a los ingresos que devengan las familias; el o $4 \%$ recibe un SMML Vy el 21\% tienen ingresos superiores a los 2 SMMLV. Estos ingresos provienen de actividades realizadas en el sector informal de la economía, tal como lo señalan en el análisis de contexto de los Proyectos Educativos Institucionales las 3 Instituciones Educativas.

\section{Gráfica No.2. Nivel de ingreso de la unidad familia del egresado}

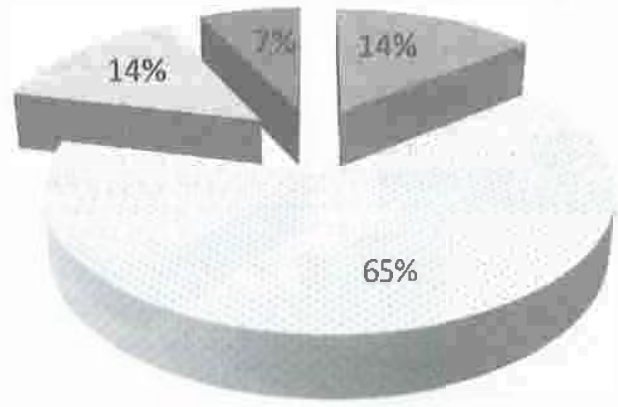

\footnotetext{
Menos de un salario mínimo

Dos salarios mínimos
}

Un salario mínimo

- Mas de tres salarios mínimo

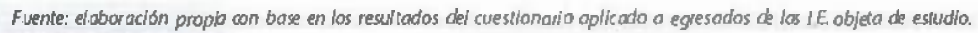

55. fuventud y trabajo en offas. Publicado of 5 de Marzo, 2010, por Porta Educativo Colombia Aprende. Recuperado el 24 dejunio de 2013 Disporible deste Internet URI: htto / / 


\section{ACTIVIDADES QUE REALIZAN LOS EGRESADOS}

Para determinar si las actividades que desarrollan los egresados están en concordancia con lo que se platea en la Política pública yen el Convenio: "Ofrecer formación técnica que proporcione posibilidades de movilidad hacia otras modalidades y niveles educativos (Cadena de formación) o para vincularse laboralmente"; se dieron a los egresados las siguientes opciones de respuesta en el cuestionario diseñado para esta investigación.

- Formación tecnológica (Cadena de formación - SENA)

- Estudios universitarios

- Estudia y trabaja de manera independiente

- Solo trabaja de manera independiente

- Estudia y trabaja como empleado

- Solo trabaja

- Desempleado

Se pudo comprobar a travês del estudio que efectivamente para esta muestra se cumpie en un 78\% el propósito de continuar con la educación superior teniendo en cuenta para este estudio la cadena de formación del SENA, señalada en los objetivos del Convenio interadministrativo 002 de 2005.

De acuerdo con las opciones planteadas se observa en Gráfica 3, la tendencia de los egresados a continuar con sus estudios superiores (teniendo en cuenta la formación tecnológica del SENA), ya sea dedicando de manera exclusiva su tiempo a esta actividad, o combinando estudio y trabajo.

Por otra parte, es importante revisar los datos de desempleo; el 12\% de los egresados se encuentran desempleados, úfra aparentemente baja si se tiene en cuenta que de los 48 egresados dedicados exclusivamente a estudiar, está buscando un empleo, pues pese a que a los estudiantes nose les califica como desempleados, su situación de precariedad en los ingresos familiares los motiva a buscar un empleo para generar ingresos y mejorar sus condiciones de vida.

Grafica 3. Actividad realizada por los egresados

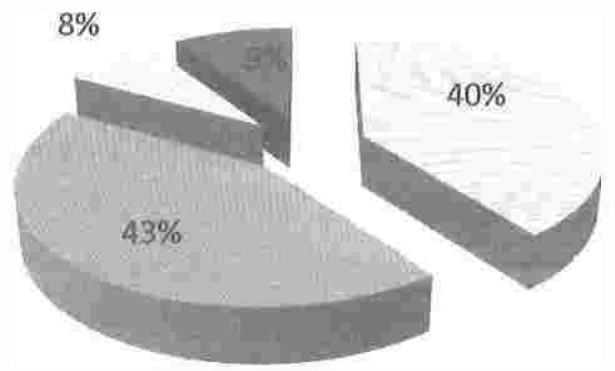

15 años 16 años 17 años 18 años 
Delos 53 egresados que continuaronla educación superior, $37(70 \%)$ se matricularon en un programa de formación tecnológica, ofertada por el SENA y el 10 ó $\left(30^{\circ} \%\right)$ se matricularon en la Universidad.

\section{INSERCIÓN LABORAL}

Uno de los propósitos del convenio es el de Propiciar la inserción de los jóvenes al trabajo. De acuerdo con el resultado de las encuestas, 11 egresados se encuentran trabajando. Al preguntarles sobre el tipo de contrato laboral, 8 respondieron "a término indefinido", los otros contratos son: uno por prestación de servicios y dos por horas.

Al cruzar la información de los 8 egresados que respondieron que tienen un contrato a término indefinido con beneficios laborales y los ingresos percibidos, se encuentra que sólo un egresado tiene todas las garantías de un contrato laboral.

Esta situación de alguna manera se refleja en el nivel de inconformismo respecto a su trabajo: al ó $9 \%$ de los egresados empleados no les agrada lo que hacen y sólo el $31 \%$ manifiesta sentirse a gusto.

Par revisar la pertinencia de las actividades laborales frente al programa de formación recibido, se preguntó a los empleadores sobre el tipo de actividad que ejecutan los egresados. Sus respuestas permiten establecer que estos egresados encontraron oportunidades laborales gracias a su formación y que si no desarrollan plenamente las competencias adquiridas, sí ejecutan actividades afines a $\mathrm{a}$ formación recibida.

Para el caso de la aplicación de las competencias específicas en el desempeño del trabajo se determina queen el caso de los egresados del área contable, no aplican directamente las competencias, ya que uno de ellos realiza labores de asistencia de dirección médica y el otro registra información en un aplicativo y factura. Estas actividades se han realizado de una forma adecuada según los empleadores, gracias a la formación recibida en el SENA.

\section{PERCEPCIÓN DE LOS ACTORES DEL PROCESO}

De las entrevistas realizadas a los funcionarios de la Secretaría de Educación, rectores y funcionarios SENA, se pueden encontrar percepciones positivas y negativas respecto al desarrollo del proceso de la articulación de la Educaciôn Media en el municipio de Neiva, se comparten algunas de ellas:

\section{Respecto a la importancia de la política:}

"Ia polficica de articuiación tiene un aspecto totaimente importante al permitirle a la Institución educativa ampliar su oferta de servicio a la comumidad, y esta of erta se manifiesta en un portafolio de opciones educativas para que los jóvenes puedan escoger y ampliar más su horizonte vocacional, bien sea para fortalecer su formación académica, o fortalecer su formación productiva, y que le permita al estudiante tener mayores opciones para escoger una u otra prof esión sobre la que más se incline o vincularse al sector productivo". 


\section{Frente a las opciones para los jóvenes:}

"Ocuparles ese tiempo muerto que ellos a veces tienen, porque lo mayoría de lo formación se hace en contra jornada. Yo rescato mucho que cuando los estudiontes tienen algo diferente a bo academico, de cierta manera ellos desarrollon otras habilidades cognitivas, ellos piensan más, analizon más, a veces hemos encontrado casos de muchachos de la modolidad ocadémica que no les va muy bien en las IFCES, pero a los de lo técnica les va mejor, entonces uno siente que esto que se está hociendo tiene resultados positivos".

\section{Algunos aspectos que se deben revisar}

"Encontramos deserción en más del 40\%, esto es una muestra de que no todas las articulaciones son pertinentes. Pero, pese a que evidenciamos eso, seguimos las proceso de articulación, hay que teneren cuenta que no es solo desarrollar un currículo pertinenteo articulado con una de las áreas, sino que implica una serie de componentes como la infraestructura, capacitación de los docentes, los laboratorios, pero las instituciones tienen unos componentes generales, no cuentan con los requerimientos técnicos que demanda un proceso de articulación. Eso hace que el proceso se veo muy limitado".

"No hay directrices ni criterios definidos entre el SENA y el municipio de Neiva, incluso entre of deportomento, que nos permita hocer una orientoción general a las instituciones educativas. En este sentido estamos definiendo unos criterios, pora que las Instituciones Educativas que se quieran articular, tengan que guiarse con unas pollticas definidas. Tenemos que parar los procesos de cobertura, evaluarlos, hacer ajustes, definir cuáles pueden seguir, cuáles mo, porque no son pertinentes."

\section{CONCLUSIONES}

\section{Las principales conclusiones que se derivan del presente trabajo de investigación son:}

Los objetivos del convenio interadministrativo 002 de 2005 se han cumplido de manera parcial, si se tiene en cuenta que: Se ha logrado la movilidad del $54 \%$ de los egresados hacia programas del nivel Tecnológico impartido por en el SENA, en la misma línea de los programas Técnicos Laborales desarrollados en las I.E.

El $25 \%$ de egresados han continuado su educación con el nivel de Pregrado de los cuales el $73 \%$ continuaron programas universitarios relacionados con la formación Técnica Laboral recibida en a I.E.

La inserción laboral de los egresados no se cumple a cabalidad dada la corta edad en la que se termina el bachiller ato en Colombia ( 15 a 18 años) y sumado a esta el deterioro de la oferta laboral del país.

No existen líneas de crëdito para iniciativas de los menores de edad y de acuerdo a las condiciones económicas de las familias estas no cuentan con recursos para apoyar los emprendimientos de sus hijos. 
Dada la corta edad en que terminan el bachillerato los jóvenes y las condiciones económicas de sus familias, se podría determinar que no existen opciones claras frente a oportunidades laborales y a la continuación de la educación superior; su única alternativa sería una educación pública gratuita o la posibilidad de continuar la formación tecnológica del SENA.

Los egresados que trabajan no cuentan con las garantías laborales que tienen los adultos, y si son contratados, los periodos de dur ación son muy cortos. Esta dinámica no es ajena a lo que ocurre en otras latitudes, los diferentes estudios realizados por el BiD, la OlT y otras organizaciones dan cuenta de la poca oportunidad para los jóvenes en el mundo laboral.

Se evidencia un compromiso por parte de las directivas de las I.E. articuladas, el cual se ve reflejado en el caso de las I.E. Olverio Lara Borrero y el Limonar, en la Misión, Visión y Objetivos de su Proyecto Educativo Institucional. En el caso de la I.E. Humberto Tafur Charry nose expresa este compromiso en su PEI.

Desde la percepción de los egresados y de los funcionarios de la Secretaría de Educación, es necesario revisar la oferta de los programas de Formación del SENA para brindar opciones a los estudiantes; una Institución Educativa con una sola formación técnica de alguna manera obliga a estudiar lo que la institución of rezca.

No se cuenta con unos lineamientos daros que orienten la oferta educativa de la Articulación con la Educación Media. La oferta obedece a la capacidad instalada ya programas que no ameriten grandes inversiones.

\section{RECOMENDACIONES}

Teniendo en cuenta los resultados y conclusiones del presente trabajo se realizan las siguientes recomendaciones:

A SENA ya la Secretaría de Educación de Neiva, realizar una revisión del proceso de ar ticulación, de tal manera que se den orientaciones claras a las Instituciones Educativas sobre qué programas ofertar de acuerdo al equipamiento tecnológico de las Instituciones. Educativas y necesidades del contexto regional y nacional.

Implementar el seguimiento a egresados de manera sistemática, para establecer el impacto de las acciones desarrolladas en el marco de la Articulación de la Educación Media.

Realizar evaluaciones periódicas del proceso de tal manera que se pueda determinar oportunamente la problemática de las bajas tasas de retención de aprendices y sus bajos niveles académicos.

Orientar a las I.E. que opten por la articulación para que sus PEI reflejen la intencionalidad de una formación enf ocada al desarrollo de competencias laborales generales y específicas. Es prioritario que las Secretarías de Educación del Huila realicen alianzas con la Universidad Surcolombiana y otras Instituciones de Educación superior, que posibiliten el acceso de los egresados de programas Técnicos a los Programas que of recen estas Instituciones. 
Los programas que el SENA oferte para la articulación deben ser para una salida ocupacional del Nivel $\mathrm{Cy}$ no $\mathrm{Ao} \mathrm{B}_{i}$ como lo establece el decreto 4906 de 2009 y teniendo en cuenta que la demanda laboral para salidas ocupacionales A o B, está relacionada con cargos donde existe un alto nivel de responsabilidad y se exige educación superior (tecnólogos o profesionales universitarios), nivel que solo $\mathbf{b}$ alcanzarían los egresados de educación superior.

En el proceso de articulación es necesario consolidar la triada, Secretaría de Educación, SENA y sector productivo, para facilitar por un lado el acercamiento de los estudiantes al mundo del trabajo en su etapa práctica y por otro que los empresarios, generen oportunidades de empleo para aquellos egresados que cumplan con la edad.

Teniendo en cuenta que para los empresarios es necesario contar no sólo con buenos técnicos, sino con empleados que tengan "don de gente", se debe dar importancia a las competencias laborales generales las cuales deben estar reflejadas en el desarrollo del currículo y deben ser responsabilidad de toda la comunidad educativa. 


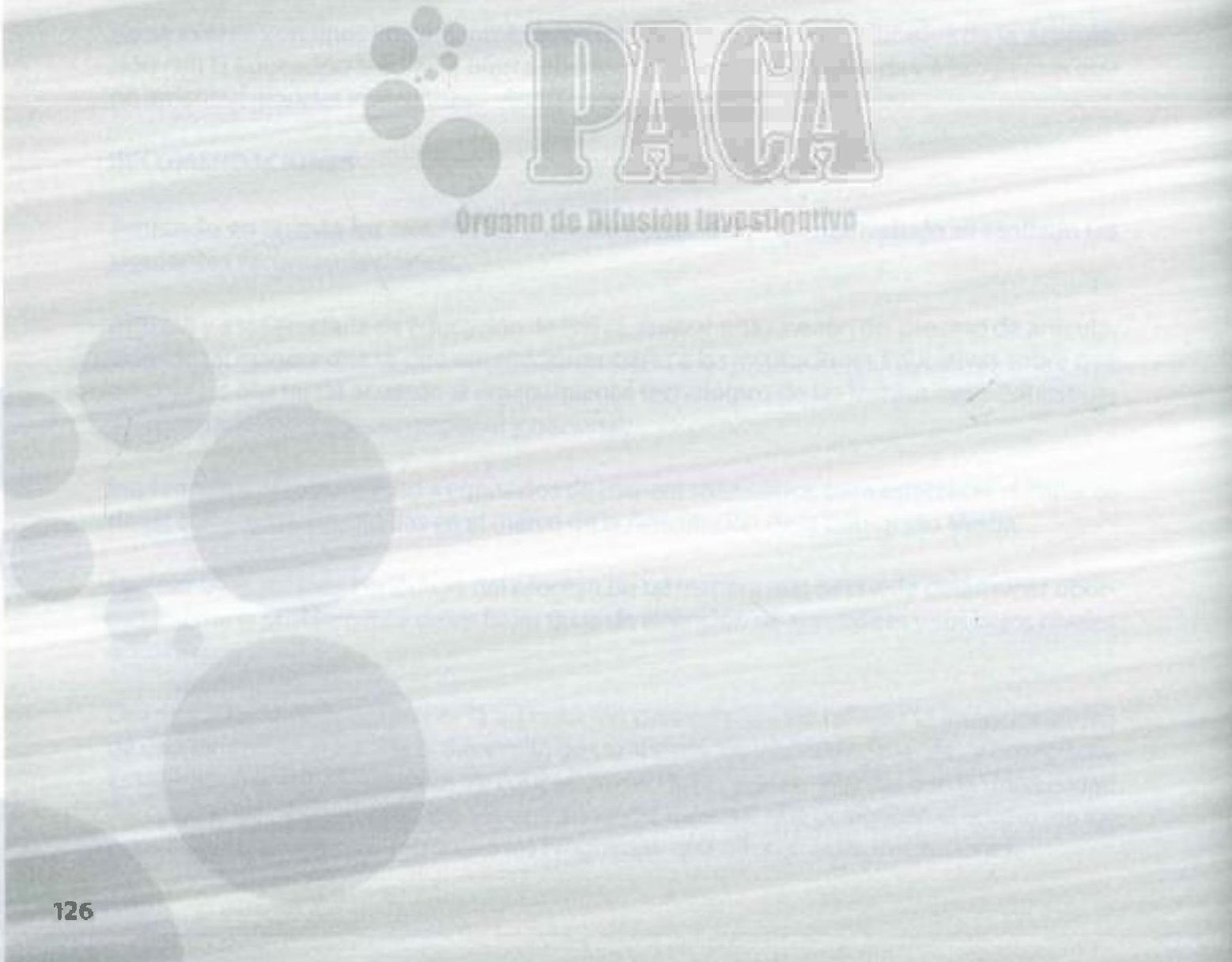

